\title{
Differences in Clinical Characteristics of Invasive Tracheobronchial Aspergillosis according to the Presence of Invasive Pulmonary Aspergillosis
}

\author{
Chuiyong Pak, M.D. ${ }^{1}{ }^{\circledR}$, Woori Jo, M.D. ${ }^{1}$, Jin Hyoung Kim, M.D. ${ }^{1}$, Jae Uk Im, M.D. ${ }^{1}$, Joseph Jeong, \\ M.D., Ph.D. ${ }^{2}$, Hee Jeong Cha, M.D., Ph.D. ${ }^{3}$, Eun-Young Choi, M.D., Ph.D. ${ }^{4}$ and Seung Won Ra, M.D., \\ Ph.D. ${ }^{1}$ (i) \\ Departments of ${ }^{1}$ Internal Medicine, ${ }^{2}$ Laboratory Medicine, and ${ }^{3}$ Pathology, Ulsan University Hospital, University of Ulsan \\ College of Medicine, Ulsan, ${ }^{4}$ Department of Internal Medicine, Yeungnam University Medical Center, University of Yeungnam \\ College of Medicine, Daegu, Republic of Korea
}

Background: The association of invasive tracheobronchial aspergillosis (ITBA) with invasive pulmonary aspergillosis (IPA) is not well established. We aimed to compare clinical characteristics between patients who exhibited ITBA with IPA and those who exhibited isolated ITBA (iITBA). Additionally, the usefulness of serum or bronchial galactomannan (GM) tests in diagnosing ITBA was evaluated.

Methods: This retrospective single-center case-control study was conducted over a period of 4 years. Fifteen patients were enrolled after confirming the presence of ITBA using bronchoscopy-guided biopsy (iITBA, 7 vs. ITBA+IPA, 8). Clinical characteristics of patients and results obtained from serum or bronchial GM tests were compared between the two groups. Mortality was assessed using data collected from a 6-month follow-up period.

Results: The ITBA+IPA group showed a higher prevalence of hematologic malignancy ( $75 \%$ vs. $14 \%$, $p=0.029$ ), a greater number of patients with multiple bronchial ulcers ( $75 \%$ vs. $14 \%, \mathrm{p}=0.029)$, lower platelet counts $(63,000 / \mu \mathrm{L}$ vs. $229,000 / \mu \mathrm{L}, \mathrm{p}<0.001)$, and a mortality rate which was significantly higher $(63 \%$ vs. $0 \%, \mathrm{p}=0.026)$ than the iITBA group. In the ITBA+IPA group, 57\% of patients tested positive according to the serum GM assay, whereas in the iITBA group, all patients tested negative ( $\mathrm{p}=0.070)$. The bronchial GM level was high in both groups, but there was no significant difference between them.

Conclusion: Patients with ITBA+IPA had a greater number of hematologic malignancies with lower platelet counts and a poorer prognosis than patients diagnosed with iITBA. Findings obtained from bronchoscopy and bronchial GM tests were more useful in diagnosing ITBA than the serum GM test results.

Keywords: Aspergillosis; Galactomannan; Bronchoscopy; Mortality

Address for correspondence: Seung Won Ra, M.D., Ph.D.

Department of Internal Medicine, Ulsan University Hospital, University of Ulsan College of Medicine, 877 Bangeojinsunhwan-doro, Dong-gu, Ulsan 44033, Republic of Korea

Phone: 82-52-250-7029, Fax: 82-52-250-7048, E-mail: docra@uuh.ulsan.kr

Received: Feb. 15, 2021, Revised: Jun. 8, 2021, Accepted: Jun. 23, 2021, Published online: Jun. 24, 2021

(a) It is identical to the Creative Commons Attribution Non-Commercial License (http://creativecommons.org/licenses/by-nc/4.0/). 


\section{Introduction}

Airborne Aspergillus spores are dispersed in the atmosphere and are small enough $(2-3 \mu \mathrm{m})$ to be easily inhaled into the peripheral airways ${ }^{1,2}$. During construction and indoor renovation, there is an increase in indoor and outdoor fungal contaminations $^{3,4}$. Inhaled spores result in various Aspergillus-related lung diseases based on different host-dependent anatomical and immunological factors, including saprophytic infections, allergic pulmonary diseases, invasive diseases, and toxic reactions $\mathrm{s}^{5}$. Invasive aspergillosis usually develops in immunocompromised hosts, such as in individuals with hematologic malignancies and neutropenia ${ }^{6}$.

Invasive tracheobronchial aspergillosis (ITBA) is a rare clinical form of invasive aspergillosis, in which the infection is primarily limited to the tracheobronchial tree ${ }^{7}$. Fungal infection has been known to be common in patients with immune suppression. However, ITBA has also been reported recently in relatively immunocompetent patients with chronic obstructive pulmonary disease (COPD), diabetes mellitus (DM), influenza and in patients admitted to the intensive care unit with non-neutropenia ${ }^{8-12}$. As a result, interest in ITBA is increasing, however its pathophysiology and association with invasive pulmonary aspergillosis (IPA), another common form of invasive aspergillosis, has not been well established. ITBA co-exists with IPA; however, in some cases, it exists without IPA. In previous research, such cases have been defined as isolated ITBA (iITBA) $)^{13}$.

Although the serum galactomannan (GM) assay is useful in diagnosing various Aspergillus-related diseases, it has limited benefits over the bronchial lavage GM assay for patients with ITBA $^{14}$. Furthermore, it is known that chest imaging is not a useful for detecting iITBA without lung parenchymal abnormalities.

Based on previous findings, we aimed to compare the clinical characteristics between patients who exhibited ITBA with IPA and those who exhibited iITBA. Additionally, the usefulness of the serum and bronchial GM assays was evaluated.

\section{Materials and Methods}

In the present study, we used the clinical data warehouse appliance (uICE, Ulsan University Hospital Information of Clinical Ecosystem) in connection with the electronic medical records of Ulsan University Hospital (UUH). We enrolled patients consecutively who were diagnosed with histologically confirmed ITBA based on the examination of one or more endobronchial lesions via bronchoscopic biopsy in the department of internal medicine of UUH between January 2008 and December 2011. From 2008 to 2011, construction of new buildings occurred at UUH, and the study period was therefore set based on the fact that aspergillosis infections had increased in the hospital during that period. This retrospective study was approved by the UUH institutional review board (IRB) and the requirement for informed consent was waived due to minimal risk of harm to the study subjects (IRB No. UUH 2021-02-015).

The presence of IPA was determined according to the European Organization for the Research and Treatment of Cancer/Mycosis Study Group (EORTC/MSG) criteria ${ }^{15}$. Patients with host risk factors, suspicious computed tomography (CT) results, and mycological proof were diagnosed with possible IPA. ITBA was defined according to the EORTC definition, which was based on the presence of tracheobronchial lesions observed during bronchoscopy and the presence of pathologic findings such as endobronchial tissue invasion and damage by Aspergillus hyphae ${ }^{15}$. Unlike IPA, ITBA cannot be diagnosed using non-invasive tests such as CT, necessitating bronchoscopy accompanied by biopsies which makes diagnosis difficult without clinical suspicion. According to bronchoscopic and pathologic findings, ITBA can be divided into three types: ulcerative, pseudomembranous, and obstructive $\mathrm{e}^{16-18}$. According to another classification, iITBA can be divided into four types: superficial infiltration (type I); full-layer involvement (type II); occlusion (type III); and mixed (type IV) types ${ }^{12,13}$. In this study, patients with ITBA and IPA were assigned to the ITBA+IPA group, and patients with ITBA without IPA were assigned to the iITBA group. Bronchoscopy-guided biopsy with bronchial washing was performed by experienced pulmonologists after identifying the presence of tracheobronchial lesions. Mucosal lesions present in patients with ITBA were classified into four different types based on the bronchoscopic appearance of intraluminal lesions ${ }^{13}$.

Data on clinical characteristics of the patients (i.e., age, sex, and underlying disease), laboratory (including culture results and GM assay) and radiologic findings were retrospectively collected from their medical records. In addition, information regarding specific respiratory symptoms or signs was collected. Six-month mortality rate was assessed using follow-up data.

The GM assay was performed using Platelia Aspergillus antigen enzyme-linked immunosorbent assay (Bio-Rad Laboratories, Marnes-la-Coquette, France) and photometric detection (STRATEC Biomedical AG Gemini Combo, BirkenfeldGrafenhausen, Germany) at $450 \mathrm{~nm}$. Indices (optical density [OD] of the sample/OD of the cutoff control) of $\geq 0.4$ were considered as positive ${ }^{19}$.

Clinical characteristics of patients and results obtained from serum and bronchial GM assays were compared between the two groups (iITBA vs. ITBA+IPA). Proportions were compared using the chi-squared test or the Fisher exact test. Considering that the number of samples is small, the Mann-Whitney U test was used for continuous variables. A $p$-value of $<0.05$ was considered to be statistically significant. The data were analyzed using the SPSS software version 24 (IBM Corp., Armonk, NY, USA). 


\section{Results}

Fifteen patients who satisfied our criteria were identified using uICE based on the presence of Aspergillus infection, which was histologically confirmed via bronchoscopic biopsy between January 2008 and December 2011. Among them, eight were diagnosed with IPA based on chest CT reports (iITBA, 7 vs. ITBA+IPA, 8). ITBA classification according to the endobronchial lesion type was as follows: type I, 11 patients; type II, 1 patient; type III, 0 patients; and type IV, 3 patients.

There were no statistically significant differences in baseline characteristics such as age, sex, and symptoms between the two groups (Table 1). The ITBA+IPA group showed a higher prevalence of hematologic malignancy $(75 \%[\mathrm{n}=6]$ vs. $14 \%$ $[n=1], p=0.029$ ) than the iITBA group. Other underlying diseases included solid tumors, systemic lupus erythematosus with systemic corticosteroid, chronic liver disease, DM, and chronic lung disease.

With regard to laboratory findings, white blood cell counts and C-reactive protein levels were not significantly different between the groups, however platelet counts were lower in the ITBA+IPA group than in the iITBA group $(63,000 / \mu \mathrm{L}$ vs.

Table 1. Baseline characteristics of patients with isolated invasive tracheobronchial aspergillosis and invasive tracheobronchial aspergillosis with invasive pulmonary aspergillosis

\begin{tabular}{|c|c|c|c|}
\hline & Isolated ITBA $(\mathrm{n}=7)$ & ITBA with IPA (n=8) & p-value* \\
\hline Age, yr & $62(42-73)$ & $58(46-72)$ & 0.536 \\
\hline Male sex & $4(57)$ & $6(75)$ & 0.608 \\
\hline \multicolumn{4}{|l|}{ Underlying diseases } \\
\hline Hematologic malignancy & $1(14)$ & $6(75)$ & 0.029 \\
\hline \multicolumn{4}{|l|}{ Non-hematologic disease } \\
\hline Solid tumor & 3 & 0 & \\
\hline SLE & 2 & 0 & \\
\hline Chronic liver disease & 2 & 2 & \\
\hline $\mathrm{DM}$ & 4 & 1 & \\
\hline Chronic lung diseases & 5 & 0 & \\
\hline \multicolumn{4}{|l|}{ Symptoms } \\
\hline Cough/sputum & $4 / 5$ & $3 / 2$ & NA \\
\hline Fever & $4(57)$ & $4(50)$ & \\
\hline Dyspnea & $2(29)$ & $3(38)$ & \\
\hline \multicolumn{4}{|l|}{ Laboratory finding } \\
\hline WBC & $8,620(280-19,100)$ & $1,935(60-17,970)$ & 0.281 \\
\hline ANC $<500$ & 1 & 3 & \\
\hline Platelet count $\left(\times 10^{3}\right)$ & $229(155-487)$ & $63(22-118)$ & $<0.001$ \\
\hline $\mathrm{CRP}(\mathrm{mg} / \mathrm{dL})$ & $3.54(0.09-22.34)$ & $17.19(2.51-25.75)$ & 0.281 \\
\hline \multicolumn{4}{|l|}{ Bronchoscopic finding } \\
\hline Presence of multiple ulcers & $1 / 7(14)$ & 6/8 (75) & 0.029 \\
\hline Type I/II/III/IV & $5 / 1 / 0 / 1$ & $6 / 0 / 0 / 2$ & NA \\
\hline Bronchial GM positivity & $6 / 6(100)$ & 5/6 (83) & NS \\
\hline Serum GM positivity & $0 / 6(0)$ & $4 / 7(57)$ & 0.070 \\
\hline Fungal culture, $\mathrm{n}$ & A. fumigatus (4) & $\begin{array}{l}\text { A. fumigatus (3) } \\
\text { A. terreus (1) }\end{array}$ & NA \\
\hline Mortality due to aspergillosis & $0 / 7(0)$ & $5 / 8(63)$ & 0.026 \\
\hline
\end{tabular}

Values are presented as median (range) or number (\%).

*Used the chi-square test or the Fisher exact test for categorical variables and the Mann-Whitney U test for continuous variables.

ITBA: invasive tracheobronchial aspergillosis; IPA: invasive pulmonary aspergillosis; SLE: systemic lupus erythematosus; DM: diabetes mellitus; NA: not available; WBC: white blood cell; ANC: absolute neutrophil count; CRP: C-reactive protein; GM: galactomannan; NS: not significant. 
$229,000 / \mu \mathrm{L}, \mathrm{p}<0.001)$. Moreover, the number of patients with multiple bronchial ulcers was higher in the ITBA+IPA group than in the iITBA group ( $75 \%[n=6]$ vs. $14 \%[n=1], p=0.029)$ (Table 1).

In the ITBA+IPA group, 57\% of the patients tested positive according to the serum GM assay, whereas all patients with iITBA tested negative. The serum GM level was higher in the ITBA+IPA group than in the iITBA group (median [range], 0.906 [0.270-4.334] vs. $0.162[0.080-0.321] ; \mathrm{p}=0.002$ ). The bronchial GM level was high in both groups with no significant difference statistically (median [range], 2.640 [0.780-5.670] vs. 3.70 [0.296-5.294]; $\mathrm{p}=0.937$ ). A direct comparison of the serum and bronchial GM levels showed that the levels were significantly higher in the bronchial samples than in the serum samples of all patients (median [range], 3.70 [0.296-5.670] vs. 0.321 [0.080-4.334]; $\mathrm{p}=0.001$ ) (Figure 1).

From the time of ITBA diagnosis, the 6-month mortality rate was significantly higher in the ITBA+IPA group than in the iITBA group $(63 \%[\mathrm{n}=5]$ vs. $0 \%[\mathrm{n}=0], \mathrm{p}=0.026)$.

\section{Discussion}

In this study, the ITBA+IPA group showed a higher rate of hematologic malignancies with lower platelet counts, higher prevalence of multiple bronchial ulcers, and poorer prognosis than the iITBA group. The results obtained from bronchial GM assay were positive in both the iITBA and ITBA+IPA groups; however, results obtained from serum GM assay were positive only in the ITBA+IPA group. Furthermore, the serum GM level was higher in the ITBA+IPA group than in the iITBA

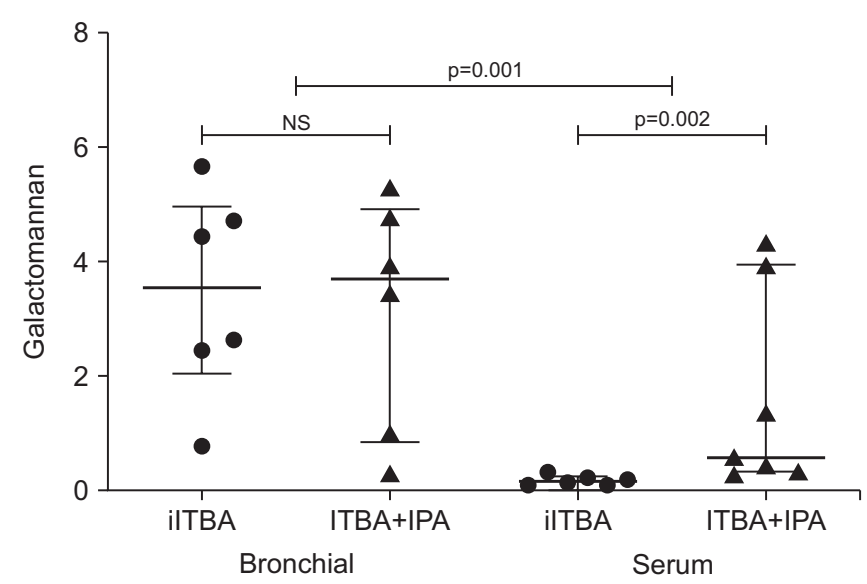

Figure 1. Bronchial and serum galactomannan levels in patients with iITBA and ITBA+IPA. The plots indicate individual data points and median \pm interquartile ranges of the mean galactomannan levels (horizontal line and vertical bar). p-value for Mann-Whitney U test $(p=0.001)$. NS: not significant; ITBA: invasive tracheobronchial aspergillosis; iITBA: isolated ITBA; IPA: invasive pulmonary aspergillosis. group.

The high proportion of patients with hematologic malignancies in the ITBA+IPA group seemed to be related to an uncontrolled hematologic disease and chemotherapy, which can cause long-lasting immune suppression without recovery. Although the temporal relationship between ITBA and IPA is unclear, it can be hypothesized that these forms may represent different stages of the same disease process and the rate of progression to IPA is high if there is a severe immune suppression in patients with ITBA ${ }^{7,20}$. Recent studies showed that the mortality rate was higher in patients with ITBA+IPA (90\%) than in those with IPA (32\%) or ITBA alone (iITBA, $22 \%)^{11,12}$. Similar results were observed in our study, which suggests that the prognosis became poorer when ITBA coexisted with IPA. Therefore, an early diagnosis and treatment of iITBA before the development of IPA are crucial. In general, multiple or extensive endobronchial lesions are thought to indicate a more advanced and severe stage of the disease than a single lesion. Higher number of bronchial ulcers found in the ITBA+IPA group might progress to ITBA+IPA as opposed to the ITBA group, in which Aspergillus infection was limited to superficial mucosa without further invasion to the bronchial tissue and vessel.

In addition to unavailability or poor interpretation of serological biomarkers, the diagnosis of ITBA without IPA was difficult and delayed because of its non-specific clinical presentation and radiological findings. By definition, the typical chest CT findings associated with IPA, such as nodules with halo signs or cavities, were not observed in patients with iITBA. It may be possible to diagnose fungal diseases in patients with immunodeficiency because they are suspected to contract opportunistic infections. However, it was more difficult in immunocompetent individuals because clinicians tend to hardly suspect it ${ }^{1,21}$. Respiratory Aspergillus infection is a well-known complication in severely and chronically immunocompromised hosts. However, mildly immunocompromised hosts, such as those with COPD, advanced liver cirrhosis, or diabetes, has been reported to be at a risk of contracting ITBA or IPA ${ }^{8-10}$. In addition, ITBA has recently been reported in patients with systemic viral infection such as influenza and severe fever thrombocytopenia syndrome ${ }^{11,21}$. Therefore, attention should be paid to whether ITBA has occurred and if suspected, bronchoscopy should be considered earlier for diagnosis and treatment.

The low platelet counts in the ITBA+IPA group make it difficult to conduct invasive tests such as percutaneous needle aspiration biopsy (PCNA) for confirming suspected IPA lung lesions. If clinicians decide that direct identification of the fungus is necessary in spite of the risk of bleeding, bronchoscopy could be a safe option for obtaining samples. Our study showed that bronchial GM levels were significantly elevated in both groups. However, serum GM was not detected in patients with iITBA and similar findings have been observed in previ- 
ous studies ${ }^{22,23}$. The low GM level in patients with ITBA was explained by the hypothesis that fungal invasion was limited to the surface layer of the airway mucosa and was not sufficient to facilitate the spreading of Aspergillus antigen into circulating blood ${ }^{24}$. In invasive bronchial aspergillosis (classically observed in patients with COPD), GM is not routinely detected in blood because neutrophils prevent translocation from the pulmonary compartment to the blood compartment ${ }^{25}$. This hypothesis was confirmed by a meta-analysis in which GM antigenemia presented low sensitivity in transplant recipients without neutropenia and more recently by Van de Groep et $\mathrm{al}^{26}$ using a study population with severe influenza. The diagnostic value of bronchoalveolar lavage (BAL) fluid GM for IPA in patients without neutropenia was observed to be superior than that of serum $\mathrm{GM}^{27}$. Additionally, for the diagnosis of IPA in critically ill patients with COPD, BAL fluid GM appeared to be more sensitive than serum GM and Aspergillus isolation from lower respiratory tract ${ }^{28}$. Based on this result, we suggest that the bronchial GM assay and related tests (including biopsy) can be more useful in diagnosing ITBA than the serum GM assay. Therefore, we recommend early detection of ITBA and iITBA using bronchoscopy and bronchial GM assay, respectively. In a recent study, the usefulness of bronchoscopy in diagnosing ITBA in patients with critical illness and no neutropenia was also evaluated ${ }^{11}$. For bronchoscopy, the sensitivity, specificity, positive and negative predictive values, and overall accuracy of changes suspected to be related to ITBA were $83.3 \%, 70.3 \%, 53.2 \%, 91.2 \%$, and $74.1 \%$, respectively. The diagnostic performance of bronchoscopy was better than that of BAL culture. Interestingly, mycetoma and halo signs were observed only in the chest CT images of $4.5 \%$ and $10.4 \%$ of the study subjects, respectively. This indicated that suspected bronchoscopic finding has diagnostic value in iITBA.

Although research on ITBA is rare, significant studies have recently been published. One of them is a multicenter, retrospective, observational study conducted by Nyga et al. ${ }^{12}$ In contrast to our study, it first diagnosed IPA in patients with severe influenza and compared differences between two groups with or without ITBA. Ninety-day mortality rates and GM concentrations in BAL fluid were higher in patients with IPA+ITBA than in patients with IPA only, which is similar to the findings of our study. Together, results of the two studies indicated that ITBA+IPA is a more advanced disease and has poorer prognosis than ITBA or IPA alone. However, considering our findings, higher mortality would be attributable to neutropenia and hematologic malignancies in the IPA+ITBA group.

Our study has several significant limitations. Because ITBA is a rare disease, the study sample was small, which may have contributed to the low statistical power in the representation of the entire ITBA population. Nevertheless, the environmental management of our hospitals was strengthened during construction and the air circulation was changed with HEPA filters in the hematology ward. Consequently, after 2012, the incidence of ITBA was further reduced, which increased the difficulty of expanding the study population ${ }^{4,29,30}$. Additionally, a selection bias may have been introduced by the retrospective single-center design of the study. All bronchoscopic examinations were not conducted by a single examiner and interpretation of findings was not conducted after agreement of several clinicians; a group of sufficiently skilled pulmonologists performed these tasks. However, a single pulmonologist independently analyzed the type of ulcers present.

Although there were some limitations to this study, it presented interesting information. First, ITBA is a relatively rare disease that has not been studied extensively. Second, ITBA was more clearly defined than previous studies, using a search strategy to find patients whose biopsies had confirmed hyphae invasion. Third, we have tried to identify the characteristics and pathogenesis of ITBA. Fourth, we have suggested additional diagnostic methods such as bronchoscopy and bronchial GM.

Based on the findings of this study, we believe that attention is needed to diagnose and treat ITBA at an appropriate time in patients with immune suppression. Moreover, bronchoscopy and related tests (including bronchial GM assay, biopsy) can be more useful than the serum GM assay for diagnosing not only iITBA but also ITBA+IPA. Additionally, in patients with hematologic malignancies showing low platelet counts, tests conducted with bronchoscopy can be a safe alternative to PCNA. Through this study, we were unable to provide sufficient explanation for the relationship between IPA and ITBA, and thus further studies are required.

\section{Authors' Contributions}

Conceptualization: Ra SW. Methodology: Jeong J, Cha HJ. Formal analysis: Pak C, Ra SW. Data curation: Im JW. Validation: Choi EY. Investigation: Jo W, Kim JH. Writing - original draft preparation: Pak C, Ra SW. Writing - review and editing: Pak C, Ra SW. Approval of final manuscript: all authors.

\section{Conflicts of Interest}

No potential conflict of interest relevant to this article was reported.

\section{Funding}

No funding to declare. 


\section{References}

1. Krenke R, Grabczak EM. Tracheobronchial manifestations of Aspergillus infections. ScientificWorldJournal 2011;11:231029.

2. Kousha M, Tadi R, Soubani AO. Pulmonary aspergillosis: a clinical review. Eur Respir Rev 2011;20:156-74.

3. Alberti C, Bouakline A, Ribaud P, Lacroix C, Rousselot P, Leblanc $\mathrm{T}$, et al. Relationship between environmental fungal contamination and the incidence of invasive aspergillosis in haematology patients. J Hosp Infect 2001;48:198-206.

4. Oren I, Haddad N, Finkelstein R, Rowe JM. Invasive pulmonary aspergillosis in neutropenic patients during hospital construction: before and after chemoprophylaxis and institution of HEPA filters. Am J Hematol 2001;66:257-62.

5. Soubani AO, Chandrasekar PH. The clinical spectrum of pulmonary aspergillosis. Chest 2002;121:1988-99.

6. Kosmidis C, Denning DW. The clinical spectrum of pulmonary aspergillosis. Thorax 2015;70:270-7.

7. Fernandez-Ruiz M, Silva JT, San-Juan R, de Dios B, GarciaLujan R, Lopez-Medrano F, et al. Aspergillus tracheobronchitis: report of 8 cases and review of the literature. Medicine (Baltimore) 2012;91:261-73.

8. Meersseman W, Vandecasteele SJ, Wilmer A, Verbeken E, Peetermans WE, Van Wijngaerden E. Invasive aspergillosis in critically ill patients without malignancy. Am J Respir Crit Care Med 2004;170:621-5.

9. Guinea J, Torres-Narbona M, Gijon P, Munoz P, Pozo F, Pelaez $\mathrm{T}$, et al. Pulmonary aspergillosis in patients with chronic obstructive pulmonary disease: incidence, risk factors, and outcome. Clin Microbiol Infect 2010;16:870-7.

10. Cho BH, Oh Y, Kang ES, Hong YJ, Jeong HW, Lee OJ, et al. Aspergillus tracheobronchitis in a mild immunocompromised host. Tuberc Respir Dis 2014;77:223-6.

11. Youssif SF, Hassan EA, Moharram AM, Farhan MA, Badary DM, Hasan AAA. Is bronchoscopic view a reliable method in diagnosis of tracheobronchial aspergillosis in critically ill non-neutropenic patients? Clin Respir J 2020;14:956-64.

12. Nyga R, Maizel J, Nseir S, Chouaki T, Milic I, Roger PA, et al. Invasive tracheobronchial aspergillosis in critically ill patients with severe influenza: a clinical trial. Am J Respir Crit Care Med 2020;202:708-16.

13. Wu N, Huang Y, Li Q, Bai C, Huang HD, Yao XP. Isolated invasive Aspergillus tracheobronchitis: a clinical study of 19 cases. Clin Microbiol Infect 2010;16:689-95.

14. Husain S, Kwak EJ, Obman A, Wagener MM, Kusne S, Stout JE, et al. Prospective assessment of Platelia Aspergillus galactomannan antigen for the diagnosis of invasive aspergillosis in lung transplant recipients. Am J Transplant 2004;4:796-802.

15. De Pauw B, Walsh TJ, Donnelly JP, Stevens DA, Edwards JE, Calandra T, et al. Revised definitions of invasive fungal disease from the European Organization for Research and Treatment of Cancer/Invasive Fungal Infections Cooperative
Group and the National Institute of Allergy and Infectious Diseases Mycoses Study Group (EORTC/MSG) Consensus Group. Clin Infect Dis 2008;46:1813-21.

16. Singh N, Husain S. Aspergillus infections after lung transplantation: clinical differences in type of transplant and implications for management. J Heart Lung Transplant 2003;22:25866.

17. Cha SI, Shin KM, Yoo SS, Jeong JY, Yoon GS, Lee SY, et al. Pseudomembranous Aspergillus tracheobronchitis in an immunocompetent patient. Tuberc Respir Dis 2008;65:400-4.

18. Park BJ, Kim YK, Kim H, Kim Y, Lee HI, Kang HM, et al. A case of endobronchial aspergillosis completely obstructing lobar bronchus. Tuberc Respir Dis 2005;59:311-4.

19. Dichtl K, Seybold U, Ormanns S, Horns H, Wagener J. Evaluation of a novel Aspergillus antigen enzyme-linked immunosorbent assay. J Clin Microbiol 2019;57:e00136-19.

20. Kramer MR, Denning DW, Marshall SE, Ross DJ, Berry G, Lewiston NJ, et al. Ulcerative tracheobronchitis after lung transplantation: a new form of invasive aspergillosis. Am Rev Respir Dis 1991;144:552-6.

21. Sakaguchi K, Koga Y, Yagi T, Nakahara T, Todani M, Fujita M, et al. Severe fever with thrombocytopenia syndrome complicated with pseudomembranous Aspergillus tracheobronchitis in a patient without apparent risk factors for invasive aspergillosis. Intern Med 2019;58:3589-92.

22. Heng SC, Chen SC, Morrissey CO, Thursky K, Manser RL, De Silva HD, et al. Clinical utility of Aspergillus galactomannan and PCR in bronchoalveolar lavage fluid for the diagnosis of invasive pulmonary aspergillosis in patients with haematological malignancies. Diagn Microbiol Infect Dis 2014;79:3227.

23. Husain S, Paterson DL, Studer SM, Crespo M, Pilewski J, Durkin M, et al. Aspergillus galactomannan antigen in the bronchoalveolar lavage fluid for the diagnosis of invasive aspergillosis in lung transplant recipients. Transplantation 2007;83:1330-6.

24. van Assen S, Bootsma GP, Verweij PE, Donnelly JP, Raemakers JM. Aspergillus tracheobronchitis after allogeneic bone marrow transplantation. Bone Marrow Transplant 2000;26:11312.

25. Pfeiffer CD, Fine JP, Safdar N. Diagnosis of invasive aspergillosis using a galactomannan assay: a meta-analysis. Clin Infect Dis 2006;42:1417-27.

26. van de Groep K, Verboom DM, van de Veerdonk FL, Haas PA, van der Poll T, Schultz MJ, et al. Detection of invasive aspergillosis in critically ill patients with influenza: the role of plasma galactomannan. Am J Respir Crit Care Med 2019;200:636-8.

27. Zhou W, Li H, Zhang Y, Huang M, He Q, Li P, et al. Diagnostic value of galactomannan antigen test in serum and bronchoalveolar lavage fluid samples from patients with nonneutropenic invasive pulmonary aspergillosis. J Clin Microbiol 2017;55:2153-61.

28. He H, Ding L, Sun B, Li F, Zhan Q. Role of galactomannan 
determinations in bronchoalveolar lavage fluid samples from critically ill patients with chronic obstructive pulmonary disease for the diagnosis of invasive pulmonary aspergillosis: a prospective study. Crit Care 2012;16:R138.

29. Chang CC, Athan E, Morrissey CO, Slavin MA. Preventing invasive fungal infection during hospital building works. Intern
Med J 2008;38:538-41.

30. Talento AF, Fitzgerald M, Redington B, O'Sullivan N, Fenelon $\mathrm{L}$, Rogers TR. Prevention of healthcare-associated invasive aspergillosis during hospital construction/renovation works. J Hosp Infect 2019;103:1-12. 